\title{
Quantitative Investigation of the Energy Transfer Efficiency in Organic-Inorganic Hybrid Scintillation Materials
}

\author{
Yan Sun, Masanori Koshimizu, ${ }^{*}$ Takamasa Harada, Yutaka Fujimoto, and Keisuke Asai \\ Department of Applied Chemistry, Graduate School of Engineering, Tohoku University, Sendai 980-8579, Japan
}

(Received January 12, 2016; accepted May 20, 2016)

Keywords: scintillator, sol-gel, organic-inorganic, hybrid

We analyzed the energy transfer efficiency from the polystyrene (PS) host to a fluorescent molecule, 2-(4-tert butylphenyl)-5-(4-phenylphenyl)-1,3,4-oxadiazole (b-PBD), in PS-b-PBD binary and PS-borosilicate-b-PBD ternary scintillation materials. The Förster radius was estimated on the basis of the absorption and emission spectra of b-PBD and PS, respectively. In addition, the energy transfer efficiency was analyzed using a phenomenological model and the emission intensity from the PS host. Consistent results were obtained by these two approaches. The analysis of the energy transfer process, in combination with the analysis of concentration quenching, leads to an estimation of the optimum b-PBD concentration. The estimated concentration was in good agreement with the actual optimum concentration confirmed by measuring radioluminescence spectra. These results indicate that the analysis of the energy transfer process described in this paper is an effective way to estimate the optimum fluor concentration even for ternary scintillation materials with nanoscale structures.

\section{Introduction}

Recently, some groups, including ours, have proposed the preparation of organic-inorganic hybrid scintillation materials using the sol-gel method or other methods. ${ }^{(1-6)}$ These scintillation materials have transparent, non-hygroscopic, crack-free, and elastic monolithic properties. Furthermore, it is possible to improve detection efficiency for high-energy photons or thermal neutrons by incorporating appropriate elements or nuclides in the inorganic part while maintaining the fast response of an organic fluor.

In such scintillation materials, an organic fluor is excited via energy transfer from the host matrix, and scintillation subsequently occurs when the excited fluor returns to the ground state. Thus, scintillation performance, in particular light yield, depends strongly on the energy transfer process. For the purpose of achieving maximum energy transfer efficiency, an effective method to determine the optimum compositions of hybrid scintillators is necessary. In this study, we analyzed the energy transfer process for typical plastic scintillators [polystyrene (PS)-fluor binary systems] and for organic-inorganic hybrid scintillation materials (PS-borosilicate-fluor ternary systems).

"Corresponding author: e-mail: koshi@qpc.che.tohoku.ac.jp 


\section{Experimental Methods}

The samples were prepared using the sol-gel method. Tetramethylorthosilicate (TMOS) as a $\mathrm{SiO}_{2}$ source and trimethylborate $(\mathrm{TMB})$ as a $\mathrm{B}_{2} \mathrm{O}_{3}$ source were used. The polystyrene (PS, SigmaAldrich Co., $\mathrm{Mn}=170000)$ and fluor molecules [2-(4-tert butylphenyl)-5-(4-phenylphenyl)1,3,4-oxadiazole (b-PBD), Tokyo Kasei Kogyo Co., Ltd.] were dissolved in a solution of N,Ndimethylformamide (DMF), tetrahydrofuran (THF), and $\mathrm{H}_{2} \mathrm{O}$. Subsequently, TMB (Sigma-Aldrich Co.) and TMOS (Shin-Etsu Chemical) were added to the mixture. The mixture was vigorously stirred at $318 \mathrm{~K}$ in a thermostatic chamber for $5 \mathrm{~h}$. The resulting clear solutions were sealed in plastic containers covered tightly with aluminum foil and were maintained at 333 or $343 \mathrm{~K}$ for two weeks for gelation. After gelation, several pinholes were made in the aluminum foil, and the transparent samples were obtained by slowly drying the aerogel up to $363 \mathrm{~K}$ (heating rate: $1 \mathrm{~K} /$ h). The PS content was approximately $24 \mathrm{~mol} \%$ in the final samples. PS-fluor binary samples were prepared without TMOS or TMB in an analogous procedure. The samples were measured without any further treatment. Some of the samples exhibited spectra or luminescence intensity significantly deviated from the trend, although all the samples were fabricated in the same manner. The deviation is possibly owing to the difference in the thermal history in the drying oven, leading to a difference in the properties of b-PBD. The difference cannot be analyzed with measurements other than luminescence measurements. Hence, the data for the deviated samples are not excluded in this paper. Fluorescence and excitation spectra were recorded with a spectrofluorometer (F4500, Hitachi). Radioluminescence measurements were performed with X-ray irradiation at room temperature using an X-ray tube (target: $\mathrm{Cu}$ ) operating at $40 \mathrm{kV}$ and $40 \mathrm{~mA}$. The detection system was composed of a multichannel photon detector (MCPD-3000, Otsuka Electronics) operating at 200-700 nm. Samples of the same size $\left(5 \times 5 \mathrm{~mm}^{2} \times 1 \mathrm{~mm}\right.$ thick $)$ were located at the same place to allow direct comparison of light yield.

\section{Results and Discussion}

\subsection{Energy transfer in the b-PBD-PS binary system}

To investigate the energy transfer from PS to the organic fluor (b-PBD), we used Förster resonance energy transfer (FRET) theory. FRET is a mechanism describing energy transfer between two different fluors, a donor (D) and an acceptor (A). ${ }^{(7-10)}$ A donor (D), initially in its electronic excited state, may transfer energy to an acceptor through nonradiative dipole-dipole coupling. The essential requirement for effective transfer is that the fluorescence emission spectrum of D and the absorption or excitation spectrum of A should overlap. Förster showed that the rate of the FRET process $\left(K_{\mathrm{T}}\right)$ depends on the inverse sixth power of the distance between the $\mathrm{D}$ and A pair $(R)^{(11,12)}$ given by

$$
K_{\mathrm{T}}=\frac{1}{\tau_{\mathrm{D}}} \times\left(\frac{R_{0}}{R}\right)^{6}
$$

where $K_{\mathrm{T}}$ is the efficiency of FRET, defined as the number of energy transfer events divided by the number of photons absorbed by $\mathrm{D}$; and $\tau_{\mathrm{D}}$ is the fluorescence lifetime of $\mathrm{D}$ (i.e. the measured lifetime of $\mathrm{D}$ in the absence of $\mathrm{A}$ ), $R$ is the distance between $\mathrm{D}$ and $\mathrm{A}$, and $R_{0}$ is so-called Förster 
radius, at which distance the energy transfer rate is equal to the intrinsic decay rate of the excited states of $\mathrm{D}$. The energy transfer at this distance between $\mathrm{D}$ and $\mathrm{A}$ is considered to be $50 \% . R_{0}$ can be calculated by the overlap integral of the emission spectrum of $\mathrm{D}$ with the absorption spectrum of A and their mutual molecular orientation as expressed by the following equation: ${ }^{(13,14)}$

$$
R_{0}^{6}=\frac{9000 \kappa^{2} Q_{\mathrm{y}} \ln 10}{128 \pi^{5} n^{4} N} \int F_{\mathrm{D}}(\lambda) \varepsilon_{\mathrm{A}}(\lambda) \lambda^{4} d \lambda
$$

where $Q_{\mathrm{y}}$ is the luminescence quantum yield of the $\mathrm{D}$ in the absence of $\mathrm{A}, n$ is the refractive index of the medium, and $N$ is Avogadro's number. $F_{\mathrm{D}}(\lambda)$ is the fluorescence intensity of $\mathrm{D}$ in the wavelength range $\lambda$ to $\lambda+\Delta \lambda$ with the total intensity normalized to unity. The term $\varepsilon_{A}(\lambda)$ is the absorption coefficient of $\mathrm{A}$ at wavelength $\lambda$, and $\kappa$ is a factor describing the relative orientation of the transition dipoles of $\mathrm{D}$ and $\mathrm{A}$. The term $\kappa^{2}$ is usually assumed to be equal to $2 / 3,{ }^{(15,16)}$ which is appropriate for dynamic random averaging of $\mathrm{D}$ and $\mathrm{A}$. In this study, the energy transfer from PS to b-PBD was examined. Before calculating the Förster radius based on FRET theory, the following two assumptions were made: (i) the orientations of $\mathrm{D}$ and $\mathrm{A}$ are random and (ii) the rotation speeds of $\mathrm{D}$ and $\mathrm{A}$ are slower than the energy transfer. The quantum yield of the luminescence of PS is estimated to be 0.02 without b-PBD.(17) The refractive index of PS is 1.6. ${ }^{(18)}$ Figure 1 shows the absorption spectrum of b-PBD in DMF and the emission spectrum of PS. Based on these data and spectra, the Förster radius is calculated to be $5.5 \mathrm{~nm}$ using Eq. (2).

The energy transfer may also be discussed on the basis of the fluorescence intensity of PS as a function of b-PBD content. The excitation spectra of the samples with varying b-PBD content are shown in Fig. 2, where the emission wavelength was set at $326 \mathrm{~nm}$ corresponding to the luminescence from PS. ${ }^{(17)}$ A peak was observed at 250-280 nm without b-PBD. Thus, this peak may be ascribed to the excitation of the PS matrix, which is consistent with the wavelength of absorption of PS at $269 \mathrm{~nm} .{ }^{(17)}$ The intensity in the excitation spectra at $269 \mathrm{~nm}$ as a function of b-PBD content is displayed in Fig. 3. The intensity was found to decrease with increasing

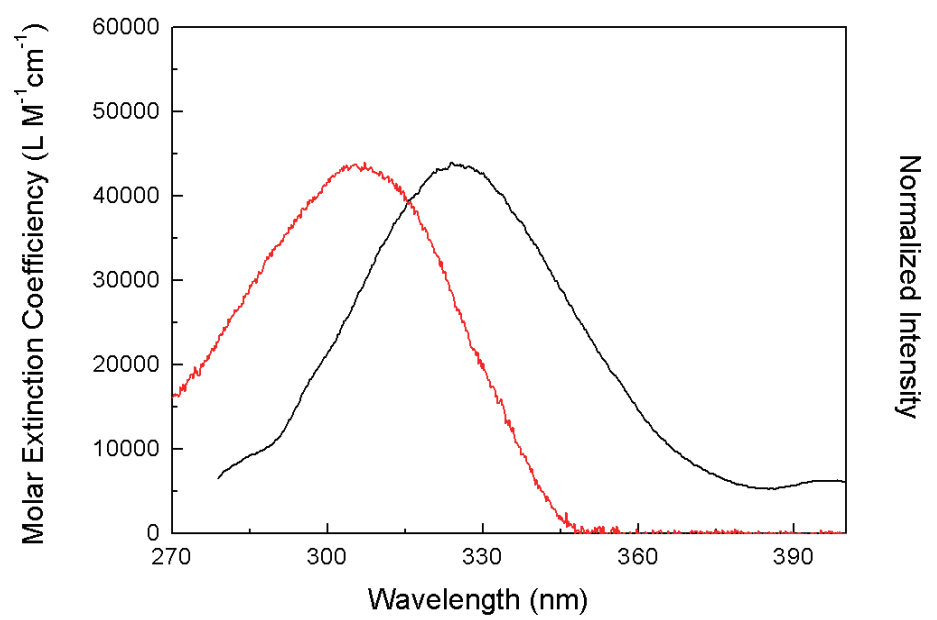

Fig. 1. (Color online) Absorption spectrum of b-PBD (red line) in DMF solution and emission spectrum of PS (black line). 


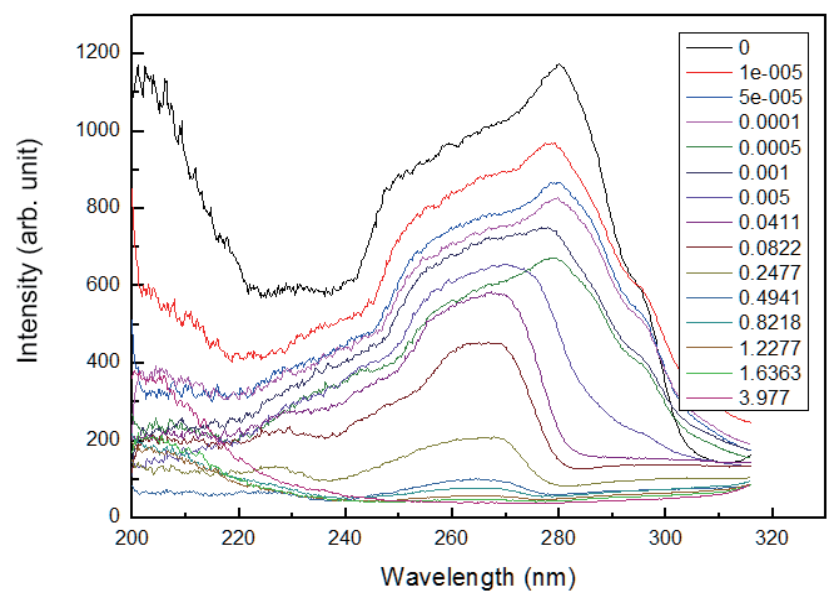

Fig. 2. (Color online) Excitation spectra of PS samples with various b-PBD concentrations $\left(\lambda_{\mathrm{em}}\right.$ $=326 \mathrm{~nm}$ ). The legends represent the b-PBD concentrations in $\mathrm{mol} \%$.

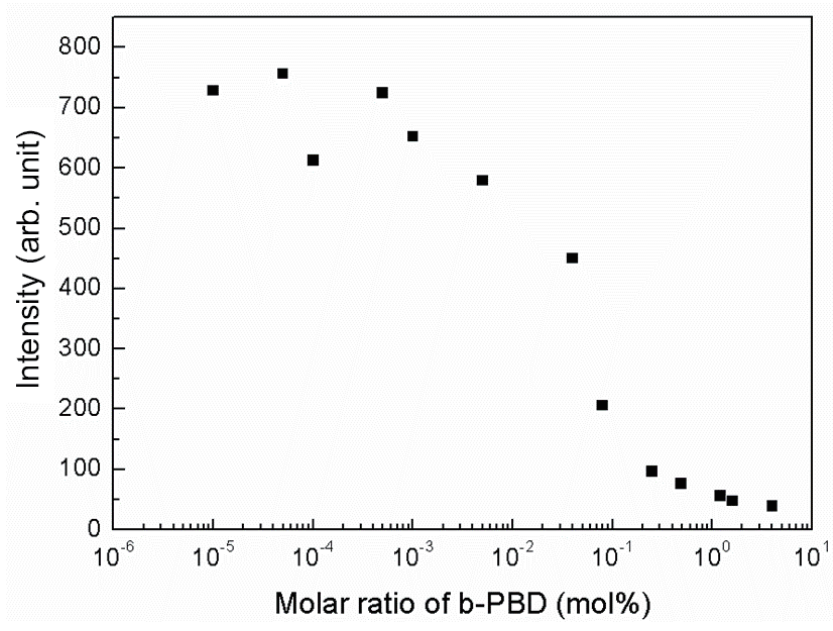

Fig. 3. Intensity of excitation spectra of PS samples at $269 \mathrm{~nm}$ as a function of b-PBD concentration.

b-PBD content. These results show that energy transfer from PS to b-PBD occurs. However, excitation light at $269 \mathrm{~nm}$ was also absorbed by b-PBD. Thus, it is not possible to ignore the reduced absorption of the excitation light by PS due to the absorption of b-PBD. For this reason, the reduction in intensity at $269 \mathrm{~nm}$ in the excitation spectra can be attributed to the following processes: (i) energy transfer from PS to b-PBD; and (ii) the absorption of the excitation light by b-PBD. On the basis of these factors, the energy transfer efficiency from PS to b-PBD may be quantitatively analyzed. To simplify the model, the acceptor (b-PBD) molecules were assumed to be homogeneously distributed in the donor (PS matrix), so that the distance between b-PBD and PS was constant.

The energy transfer efficiency can be described using fluorescence intensities of D as: ${ }^{(19)}$

$$
K_{\mathrm{T}}=1-\frac{F_{\mathrm{D}}}{F_{\mathrm{D}}^{0}},
$$

where $F_{\mathrm{D}}$ and $F_{\mathrm{D}}^{0}$ are the fluorescence intensities of $\mathrm{D}$ in the presence and absence of A, respectively. When the sample is excited at $269 \mathrm{~nm}$, the absorption of b-PBD molecules cannot be ignored. Therefore, the fluorescence in the presence and absence of b-PBD molecules was calculated by the following method. Figure 4 shows schematics of the model. The total flux of the excitation light was labeled $w+x$. For the PS-only system, all flux was absorbed by PS. For PS-b-PBD systems, the absorbed flux of PS was reduced to $w$. The absorbed flux of b-PBD is denoted as $x$. The ratio of these two fluxes is calculated by the following equations:

$$
\begin{aligned}
& \frac{w}{x}=\frac{\varepsilon_{\mathrm{PS}} n_{\mathrm{PS}}}{\varepsilon_{\mathrm{b}-\mathrm{PBD}} n_{\mathrm{b}-\mathrm{PBD}}}, \\
& w=\frac{\varepsilon_{\mathrm{PS}} n_{\mathrm{PS}}}{\varepsilon_{\mathrm{b}-\mathrm{PBD}} n_{\mathrm{b}-\mathrm{PBD}}} x,
\end{aligned}
$$



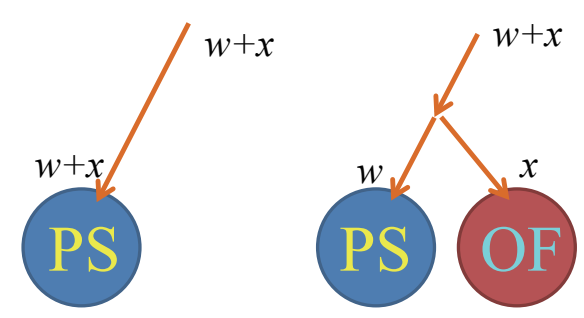

Fig. 4. (Color online) Model for considering the change in the absorption of the excitation light. PS: polystyrene; OF: organic fluor.

where, $\varepsilon$ symbolizes the molar absorption coefficients, and $n$, the number of moles. The ratio of the absorbed light flux of PS was obtained as

$$
\frac{w}{w+x}=\frac{\varepsilon_{\mathrm{PS}} n_{\mathrm{PS}}}{\varepsilon_{\mathrm{b}-\mathrm{PBD}} n_{\mathrm{b}-\mathrm{PBD}}+\varepsilon_{\mathrm{PS}} n_{\mathrm{PS}}} .
$$

In addition, based on the consideration of Fig. 4 , the ratio of $F_{\mathrm{D}}$ to $F_{\mathrm{D}}^{0}$ is calculated to be

$$
\frac{F_{\mathrm{D}}}{F_{\mathrm{D}}^{0}}=\frac{w+x}{w} \frac{F_{\mathrm{PS}}}{F_{\mathrm{PS}}^{0}}
$$

where $F_{\mathrm{PS}}$ and $F_{\mathrm{PS}}^{0}$ represent the fluorescence intensities in the presence and absence of b-PBD, respectively. The combination of Eqs. (3), (6), and (7) gives

$$
K_{\mathrm{T}}=1-\frac{w+x}{w} \frac{F_{\mathrm{PS}}}{F_{\mathrm{PS}}^{0}}=1-\left(1+\frac{\varepsilon_{\mathrm{b}-\mathrm{PBD}} n_{\mathrm{b}-\mathrm{PBD}}}{\varepsilon_{\mathrm{PS}} n_{\mathrm{PS}}}\right) \frac{F_{\mathrm{PS}}}{F_{\mathrm{PS}}^{0}} .
$$

From Eq. (8), the energy transfer efficiency can be calculated. The estimated energy transfer efficiency as a function of b-PBD content is shown in Fig. 5. The energy transfer efficiency was enhanced with b-PBD content and attained a saturation value for concentrations of b-PBD higher than $0.5 \mathrm{~mol} \%$. From the viewpoint of energy transfer only, the maximum scintillation light yield can be obtained when b-PBD is more than $0.5 \mathrm{~mol} \%$.

To confirm the applicability of the FRET theory for this system, the Förster radius was estimated based on Fig. 5. The 50\% energy transfer efficiency was estimated at $0.1 \mathrm{~mol} \%$ b-PBD based on Fig. 5 . Taking into account the density of PS of $1.04 \mathrm{~g} / \mathrm{cm}^{3}$, one b-PBD molecule takes up $1.7 \times 10^{2} \mathrm{~nm}^{3}$ of PS volume. Assuming that b-PBD is homogeneously distributed in the PS matrix, one b-PBD molecule should be located in the center of a PS sphere with a radius of $3.4 \mathrm{~nm}$. Therefore, the average distance of b-PBD and PS can be estimated to be $3.4 \mathrm{~nm}$. This value was quite similar to 5.5 $\mathrm{nm}$ which was calculated from the FRET model. Thus, FRET theory was applicable to quantify the energy transfer efficiency in the PS-b-PBD systems. The value of $3.4 \mathrm{~nm}$ was calculated based on the density of PS with the densely packed structure. However, around the relatively large molecules of b-PBD, it is natural to consider that PS will be packed more loosely. From this viewpoint, the obtained value underestimates the actual distance. In addition, the approach in this study is based on a static model. Actually the de-excitation at PS prior to the energy transfer should be considered, 


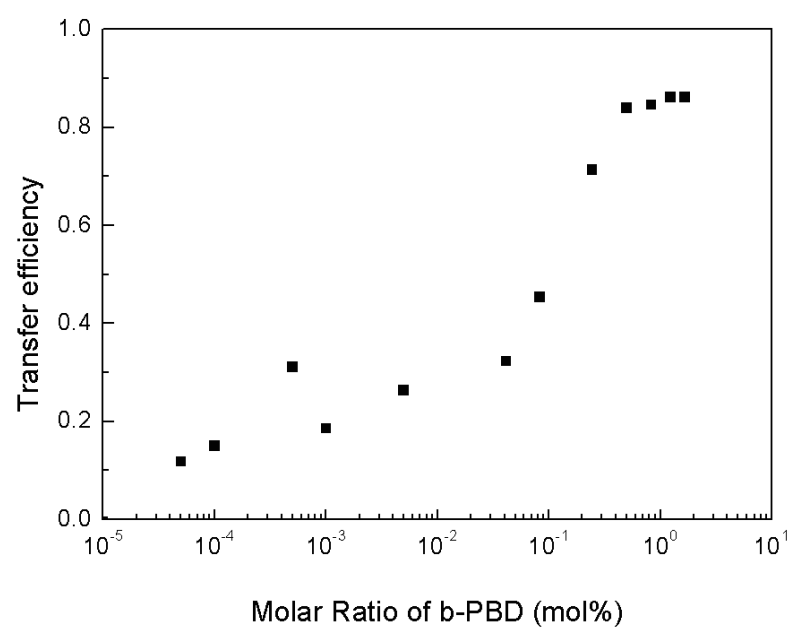

Fig. 5. Energy transfer efficiency from PS to b-PBD in PS samples as a function of b-PBD content.

which leads to a lower energy transfer efficiency and thus a lower Förster radius calculated using Eq. (2).

Considering the scintillation light yield, in addition to the energy transfer discussed above, concentration quenching must be considered. Figure 6 shows the luminescence spectra for different b-PBD contents. The excitation wavelength was set at $305 \mathrm{~nm}$, corresponding to the characteristic excitation wavelength of b-PBD. Figure 7 shows the fluorescence intensity at 367 $\mathrm{nm}$ as a function of b-PBD content. When the b-PBD concentration was less than $0.005 \mathrm{~mol} \%$, the emission intensity was enhanced by increasing b-PBD content. The intensity reached saturation when the b-PBD concentration was in the range of 0.005 to $0.5 \mathrm{~mol} \%$. The previously-mentioned concentration quenching occurred when the concentration was more than $1 \mathrm{~mol} \%$. On the basis of the results of the analysis of the energy transfer and the investigation of concentration quenching, the optimal b-PBD concentration seems to be between 0.5 and $1 \mathrm{~mol} \%$.

\subsection{Energy transfer and scintillation light yield in PS-borosilicate-b-PBD ternary systems}

The FRET theory was also applied to ternary systems. The PS content in all the samples was $24 \mathrm{~mol} \%$, and the b-PBD content was changed. In this system, the excited energy levels in PS are lower than those in borosilicate, and energy transfer between PS and borosilicate can be ignored. Hence, only energy transfer from PS to b-PBD was taken into account. Thus, a discussion similar to that in the previous section is possible. The energy transfer efficiency as a function of b-PBD concentration is displayed in Fig. 8. The energy transfer efficiency increased with b-PBD concentration. Saturation of transfer efficiency was achieved with a b-PBD content more than 0.3 mol\%. The optimal b-PBD concentration for scintillation light output was expected to occur when the $\mathrm{b}$-PBD content was more than $0.3 \mathrm{~mol} \%$ if only energy transfer was being considered.

As was stated in the previous section, concentration quenching must be considered. The luminescence spectra of PS-borosilicate hybrid samples with different b-PBD content are shown in Fig. 9. The luminescence intensity at $367 \mathrm{~nm}$ as a function of b-PBD concentration is shown in Fig. 10. The dependence for PS-borosilicate hybrid samples shown in Fig. 10 was almost the 


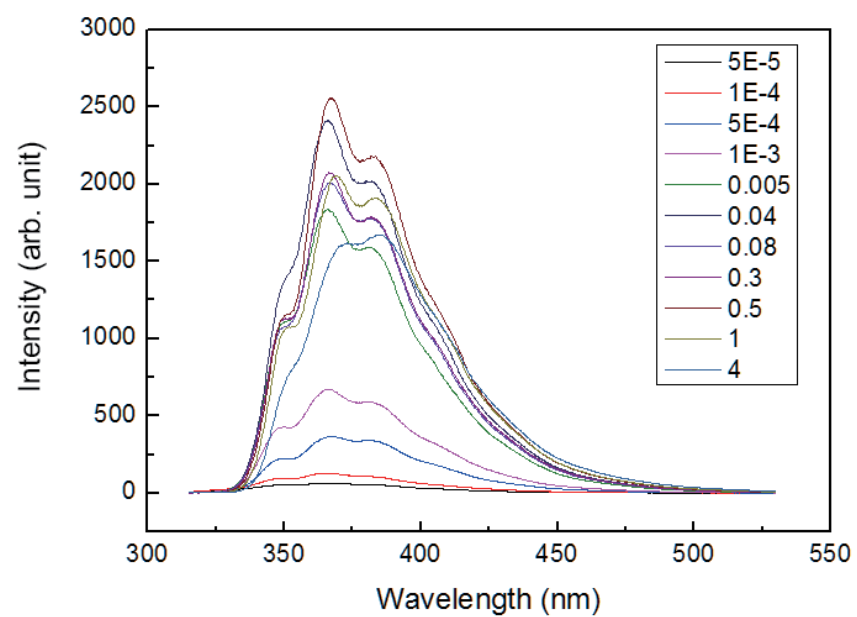

Fig. 6. (Color online) Luminescence spectra of PS samples at different b-PBD concentrations $\left(\lambda_{\mathrm{ex}}=305\right.$ $\mathrm{nm})$. The legend represents the b-PBD concentrations in $\mathrm{mol} \%$.

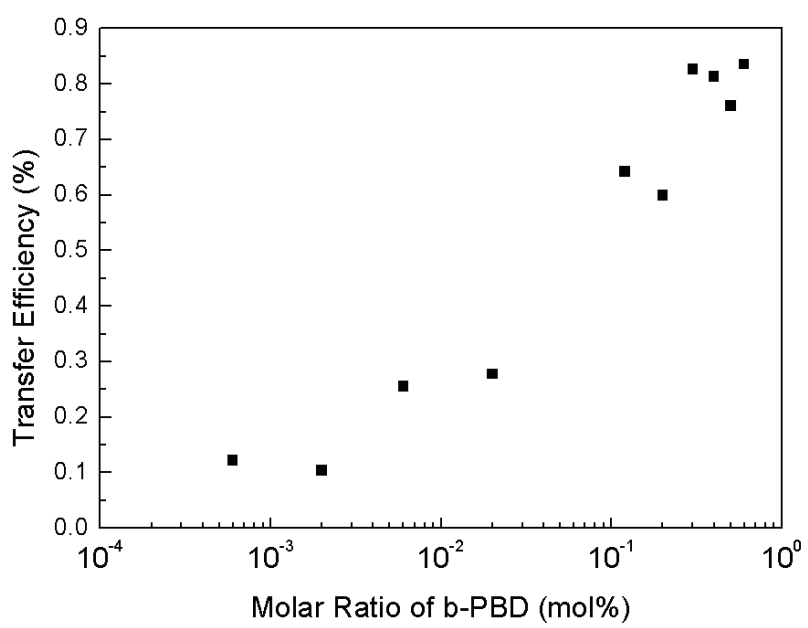

Fig. 8. Energy transfer efficiency of PS-borosilicate hybrid samples as a function of b-PBD content.

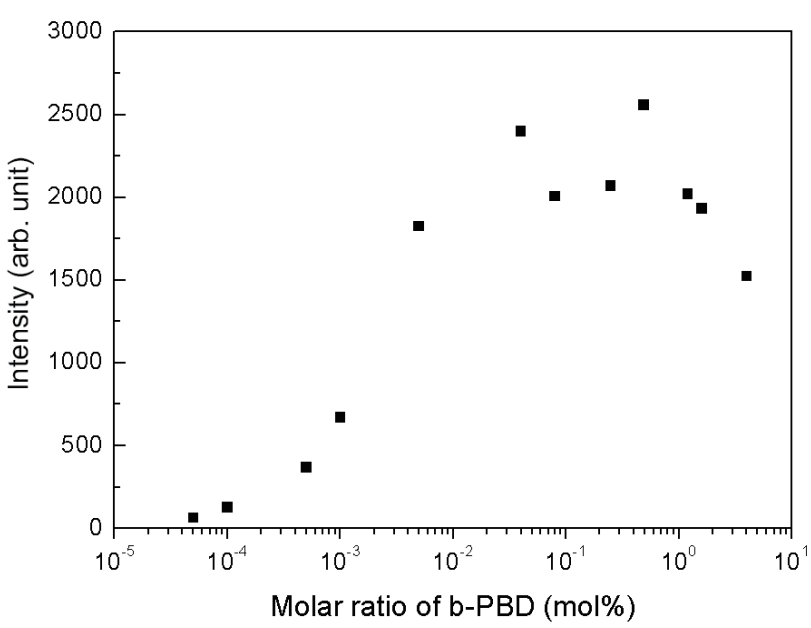

Fig. 7. Luminescence intensity at $367 \mathrm{~nm}$ for PS samples as a function of b-PBD content.

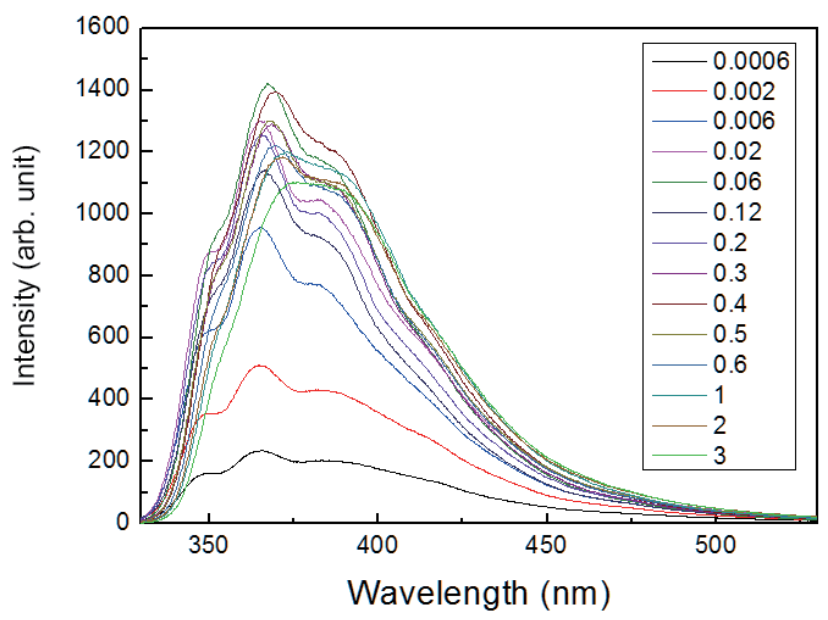

Fig. 9. (Color online) Luminescence spectra of PS-borosilicate hybrid samples at different b-PBD concentrations $\left(\lambda_{\mathrm{ex}}=305 \mathrm{~nm}\right)$. The legend represents the b-PBD concentrations in $\mathrm{mol} \%$.

same as that for the PS samples. A tendency for emission intensity to increase was observed when the b-PBD content was less than $0.02 \mathrm{~mol} \%$. In the range of 0.02 to $0.5 \mathrm{~mol} \%$, no apparent change was observed. Concentration quenching occurred beyond $0.5 \mathrm{~mol} \%$. Considering energy transfer efficiency and concentration quenching, the optimal b-PBD concentration for scintillation light yield may be expected to be approximately $0.3 \mathrm{~mol} \%$. 


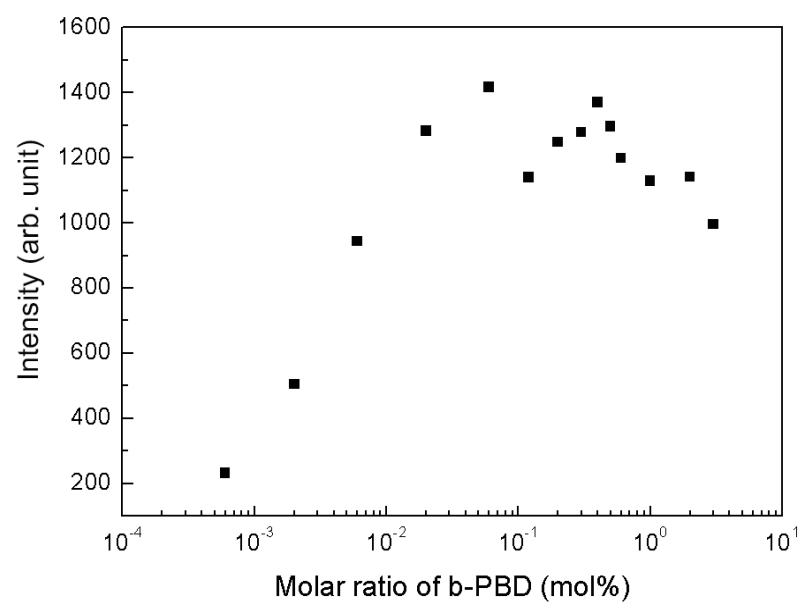

Fig. 10. Luminescence intensity at $367 \mathrm{~nm}$ for PSborosilicate hybrid samples as a function of b-PBD content.

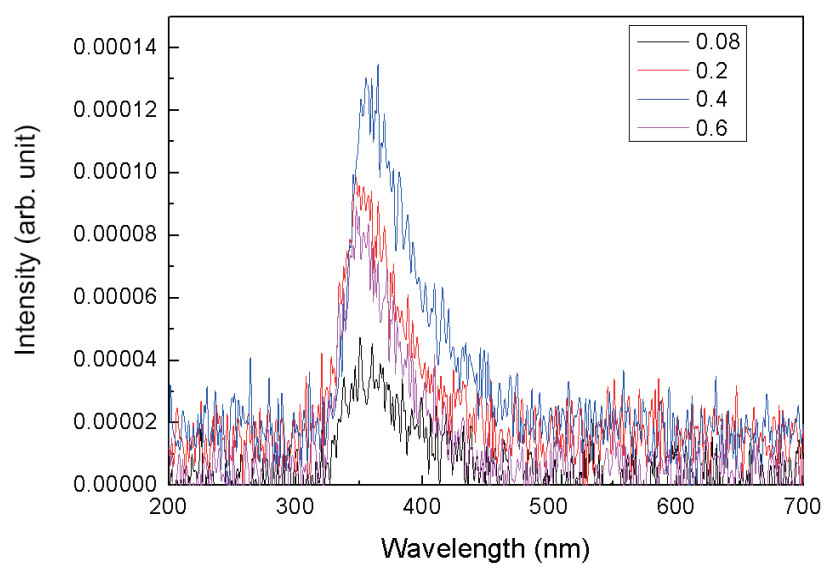

Fig. 11. (Color online) X-ray-induced radioluminescence spectra of PS-borosilicate hybrid samples at different b-PBD concentrations. The legends represent the b-PBD concentrations in $\mathrm{mol} \%$.

X-ray-induced radioluminescence spectra of PS-borosilicate hybrid samples at different b-PBD concentrations are shown in Fig. 11. The measurements were performed for the samples exhibiting efficient radioluminescence. A dominant band was observed at approximately $365 \mathrm{~nm}$, which can be ascribed to the emission from the b-PBD fluor. The $0.4 \mathrm{~mol} \%$ b-PBD-doped hybrid sample achieved the maximum scintillation light yield. On the basis of the discussion on the energy transfer, efficient scintillation is expected for the samples with b-PBD concentration larger than $0.1 \%$. On the other hand, the concentration quenching occurs for the samples with b-PBD concentration larger than $1 \%$. Thus, the maximum scintillation efficiency is expected at the b-PBD concentration between 0.1 and $1 \%$. Hence, this result is in line with our discussion of energy transfer and concentration quenching.

\section{Conclusions}

Energy transfer processes were quantitatively analyzed for the PS-b-PBD binary systems and PS-borosilicate-b-PBD ternary systems. The energy transfer efficiency was estimated using a simple phenomenological model. The energy transfer efficiency as a function of b-PBD concentration was consistent with the Förster radius estimated on the basis of the absorption and emission spectra of b-PBD and PS, respectively. These results were quite similar for the binary and ternary systems. The highest scintillation light yield was achieved for the ternary system with 0.4 mol\% b-PBD, which is in line with the analysis of energy transfer and concentration quenching. These results indicate that the quantitative analysis of the energy transfer process is a powerful tool in optimizing the fluor concentration, even in ternary scintillation materials with nanoscale structures. 


\section{Acknowledgements}

This research was partially supported by a Grant-in-Aid for Young Scientists (A) (No. 25709088, 2013-2015) and Grant-in-Aid for the Development of Systems and Technology for Advanced Measurement and Analysis from the Japan Science and Technology Agency. This work was partially supported by the Cooperative Research Project of the Research Institute of Electronics, Shizuoka University.

\section{References}

1 M. Koshimizu, H. Kitajima, T. Iwai, and K. Asai: Jpn. J. Appl. Phys. 47 (2008) 5717.

2 H.-J. Im, B. Lee, S. S. Brown, and S. Dai: J. Nanosci. Nanotechnol. 7 (2007) 3784.

3 H.-J. Im, C. Willis, S. Saengkerdsub, R. Makote, M. D. Pawel, and S. Dai: J. Sol-Gel Sci. Technol. 32 (2004) 117.

4 H.-J. Im, C. Willis, A. C. Stephan, M. D. Pawel, S. Saengkerdsub, and S. Dai: Appl. Phys. Lett. 84 (2004) 2448.

5 Y. Sun, M. Koshimizu, N. Yahaba, F. Nishikido, S. Kishimoto, R. Haruki, and K. Asai: Appl. Phys. Lett. 104 (2014) 174104.

6 Y. Araya, M. Koshimizu, R. Haruki, F. Nishikido, S. Kishimoto, and K. Asai: Sens. Mater. 27 (2015) 255.

7 P. G. Wu and L. Brand: Anal. Biochem. 218 (1994) 1.

8 J. R. Lakowicz: Principles of Fluorescence Spectroscopy (Plenum, New York, 1983) p. 305.

9 T. Förster: Naturwissenschafien 33 (1946) 166.

10 T. Förster: Fluorescence of Organic Compounds (Vandenhoeck \& Ruprecht, Göttingen, 1951) p. 158.

11 T. Förster: Discuss. Faraday Soc. 27 (1959) 7.

12 A. Dietrich, V. Buschmann, C. Müller, and M. Sauer: Rev. Mol. Biotechnol. 82 (2002) 211.

13 P. R. Selvin: Method Enzymol. 246 (1995) 300.

14 T. Förster: Annalen der Physik 437 (1948) 55.

15 I. Steinber: Annu. Rev. Biochem. 40 (1971) 83.

16 R. E. Dale, J. Eisinger, and W. E. Blumberg: Biophys. J. 26 (1979) 161.

17 F. Hirayama, L. J. Basile, and C. Kikuchi: Mol. Cryst. 4 (1968) 83.

$18 \mathrm{http}: / /$ refractiveindex.info/?group=PLASTICS\&material=PS

19 R. M. Clegg: Fluorescence Imaging Spectroscopy and Microscopy (Wiley, New York, 1996) p 179. 